\title{
Front Matter: Volume 9154
}

"Front Matter: Volume 9154," Proc. SPIE 9154, High Energy, Optical, and Infrared Detectors for Astronomy VI, 915401 (12 August 2014); doi: $10.1117 / 12.2076800$

SPIE Event: SPIE Astronomical Telescopes + Instrumentation, 2014, Montréal, Quebec, Canada 


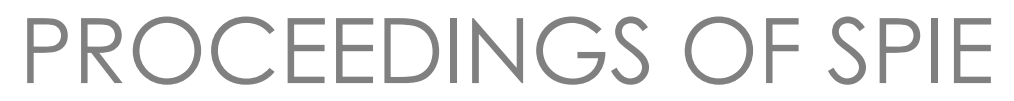

\title{
High Energy, Optical, and Infrared Detectors for Astronomy VI
}

\author{
Andrew D. Holland \\ James W. Beletic \\ Editors
}

22-25 June 2014

Montréal, Canada

Sponsored and Published by

SPIE 
The papers included in this volume were part of the technical conference cited on the cover and title page. Papers were selected and subject to review by the editors and conference program committee. Some conference presentations may not be available for publication. The papers published in these proceedings reflect the work and thoughts of the authors and are published herein as submitted. The publisher is not responsible for the validity of the information or for any outcomes resulting from reliance thereon.

Please use the following format to cite material from this book:

Author(s), "Title of Paper," in High Energy, Optical, and Infrared Detectors for Astronomy VI, edited by Andrew D. Holland, James W. Beletic, Proceedings of SPIE Vol. 9154 (SPIE, Bellingham, WA, 2014) Article CID Number.

ISSN: 0277-786X

ISBN: 9780819496225

Published by

SPIE

P.O. Box 10, Bellingham, Washington 98227-0010 USA

Telephone +1 3606763290 (Pacific Time) · Fax +1 3606471445

SPIE.org

Copyright (@ 2014, Society of Photo-Optical Instrumentation Engineers.

Copying of material in this book for internal or personal use, or for the internal or personal use of specific clients, beyond the fair use provisions granted by the U.S. Copyright Law is authorized by SPIE subject to payment of copying fees. The Transactional Reporting Service base fee for this volume is $\$ 18.00$ per article (or portion thereof), which should be paid directly to the Copyright Clearance Center (CCC), 222 Rosewood Drive, Danvers, MA 01923. Payment may also be made electronically through CCC Online at copyright.com. Other copying for republication, resale, advertising or promotion, or any form of systematic or multiple reproduction of any material in this book is prohibited except with permission in writing from the publisher. The CCC fee code is 0277-786X/14/\$18.00.

Printed in the United States of America.

Publication of record for individual papers is online in the SPIE Digital Library.

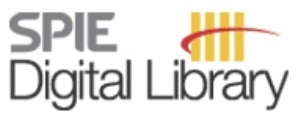

SPIEDigitalLibrary.org

Paper Numbering: Proceedings of SPIE follow an e-First publication model, with papers published first online and then in print and on CD-ROM. Papers are published as they are submitted and meet publication criteria. A unique, consistent, permanent citation identifier (CID) number is assigned to each article at the time of the first publication. Utilization of CIDs allows articles to be fully citable as soon as they are published online, and connects the same identifier to all online, print, and electronic versions of the publication. SPIE uses a six-digit CID article numbering system in which:

- The first four digits correspond to the SPIE volume number.

- The last two digits indicate publication order within the volume using a Base 36 numbering

system employing both numerals and letters. These two-number sets start with 00, 01, 02, 03, 04, $05,06,07,08,09,0 A, 0 B \ldots 0 Z$, followed by 10-1Z, 20-2Z, etc.

The CID Number appears on each page of the manuscript. The complete citation is used on the first page, and an abbreviated version on subsequent pages. Numbers in the index correspond to the last two digits of the six-digit CID Number. 


\section{Contents}

xv Conference Committee

\section{Part One}

\section{SESSION 1 PROGRAMS}

915402 CNES detector development for scientific space missions: status and roadmap for infrared detectors [9154-1]

H. Geoffray, Ctr. National d'Études Spatiales (France); O. Boulade, Commissariat à I'Energie Atomique (France); B. Fièque, SOFRADIR (France); O. Gravrand, J. Rothmann, J. P. Zanatta, Commissariat à l'Energie Atomique (France); L. Tauziède, A. Bardoux, Ctr. National d'Études Spatiales (France)

915403 European Space Agency detector development for space science: present and future activities [9154-100]

L. Duvet, M. Bavdaz, P. E. Crouzet, N. Nelms, Y. R. Nowicki-Bringuier, B. Shortt, P. Verhoeve, European Space Research and Technology Ctr. (Netherlands)

915404 Detector developments at ESO to prepare for the E-ELT era [9154-102]

M. Downing, G. Finger, D. Ives, O. Iwert, J. Kolb, S. Ramsay, European Southern Observatory (Germany)

\section{SESSION 2 RADIATION DAMAGE}

915406 The radiation environment at $\mathbf{L 2}$ as seen by Gaia [9154-30]

R. Kohley, European Space Astronomy Ctr. (Spain); C. M. Crowley, European Space Astronomy Ctr. (Spain) and HE Space Operations BV (Netherlands); P. Garé, European Space Research and Technology Ctr. (Netherlands); F. Chassat, Airbus Defence and Space Satellites (France); A. D. Short, European Space Research and Technology Ctr. (Netherlands); J. M. Martin-Fleitas, A. Mora, European Space Astronomy Ctr. (Spain) and Aurora Technology (Netherlands); A. Abreu-Aramburu, European Space Astronomy Ctr. (Spain) and Elecnor Deimos Space (Spain); T. Prod'homme, European Space Research and Technology Ctr. (Netherlands)

915407 Design and characterisation of the new CIS115 sensor for JANUS, the high resolution camera on JUICE [9154-31]

M. Soman, A. D. Holland, K. D. Stefanov, J. P. Gow, M. Leese, The Open Univ. (United Kingdom); J. Pratlong, P. Turner, e2v technologies plc (United Kingdom) 
915408 In situ trap parameter studies in CCDs for space applications [9154-32]

D. J. Hall, N. Murray, J. Gow, D. Wood, A. Holland, The Open Univ. (United Kingdom)

915409 A comparative study of charge transfer inefficiency value and trap parameter determination techniques making use of an irradiated ESA-Euclid prototype CCD [9154-33] T. Prod'homme, P. Verhoeve, European Space Agency (Netherlands); R. Kohley, European Space Agency (Spain); A. Short, N. Boudin, European Space Agency (Netherlands)

\section{SESSION $3 \quad$ EM-CCDS}

9154 OA Development of a $\mathbf{4 k} \times \mathbf{4 k}$ frame transfer electron multiplying CCD for scientific applications [9154-4]

J.-L. Gach, Lab. d'Astrophysique de Marseille, CNRS, Aix Marseille Univ. (France);

C. Carignan, Univ. of Cape Town (South Africa) and Univ. de Montréal (Canada); O. Hernandez, Univ. de Montréal (Canada) and Observatoire du Mont Mégantic (Canada); P. R. Jorden, D. Jordan, e2v technologies plc (United Kingdom); P. Balard, Lab. d'Astrophysique de Marseille, CNRS, Aix Marseille Univ. (France); P. Vallée, Univ. de Montréal (Canada); P. Amram, M. Marcelin, B. Epinat, Lab. d'Astrophysique de Marseille, CNRS, Aix Marseille Univ. (France)

9154 OB EMCCDs: $10 \mathrm{MHz}$ and beyond [9154-5]

O. Daigle, Nüvü Camēras Inc. (Canada); O. Djazovski, Canadian Space Agency (Canada); M. Francoeur, Nüvü Camēras Inc. (Canada); D. G. Laurin, Canadian Space Agency (Canada); R. Doyon, Univ. de Montréal (Canada)

9154 OC Characterization of a photon counting EMCCD for space-based high contrast imaging spectroscopy of extrasolar planets [9154-6]

A. N. Wilkins, Univ. of Maryland, College Park (United States); M. W. McElwain, NASA Goddard Space Flight Ctr. (United States); T. J. Norton, NASA Goddard Space Flight Ctr. (United States) and Univ. of Maryland, Baltimore County (United States); B. J. Rauscher, NASA Goddard Space Flight Ctr. (United States); J. F. Rothe, Universities Space Research Association (United States); M. Malatesta, The Univ. of Oklahoma (United States); G. M. Hilton, Univ. of Maryland, College Park (United States) and NASA Goddard Space Flight Ctr. (United States); J. R. Bubeck, NASA Goddard Space Flight Ctr. (United States) and Adnet Systems (United States); C. A. Grady, NASA Goddard Space Flight Ctr. (United States) and Eureka Scientific, Inc. (United States); D. J. Lindler, NASA Goddard Space Flight Ctr. (United States)

9154 OD Astronomical imaging with EMCCDs using long exposures [9154-7]

O. Daigle, Nüvü Camēras Inc. (Canada); O. Djazovski, J. Dupuis, Canadian Space Agency (Canada); R. Doyon, É. Artigau, Univ. de Montréal (Canada)

9154 OE Developments in the EM-CCD camera for OGRE [9154-8]

J. H. Tutt, R. L. McEntaffer, C. DeRoo, T. Schultz, D. M. Miles, The Univ. of lowa (United States); W. Zhang, NASA Goddard Space Flight Ctr. (United States); N. J. Murray, A. D. Holland, The Open Univ. (United Kingdom); W. Cash, T. Rogers, Univ. of Colorado at Boulder (United States); S. O'Dell, J. Gaskin, J. Kolodziejczak, NASA Marshall Space Flight Ctr. (United States); A. M. Evagora, K. Holland, D. Colebrook, XCAM Ltd. (United Kingdom) 
9154 OF Comparison of EMCCD post-processing methods for photon counting flux ranges [9154-60] N. Rousset, J. Villeneuve, J.-H. Fournier-Lupien, A. Attiaoui, G. Taillon, S. Francoeur, Ecole Polytechnique de Montréal (Canada); O. Daigle, Nüvü Camēras Inc. (Canada)

\section{SESSION $4 \quad$ X-RAY DETECTORS I}

$9154 \mathrm{OH} \quad \mathrm{X}$-ray analysis of fully depleted CCDs with small pixel size [9154-42]

I. V. Kotov, J. Haupt, Brookhaven National Lab. (United States); P. Kubánek, Institute of Physics of the ASCR, v.v.i. (Czech Republic); P. O'Connor, P. Takacs, Brookhaven National Lab. (United States)

9154 Ol Dynamic range of fully depleted pnCCDs: modeling and experimental confirmation [9154-29]

J. Schmidt, R. Hartmann, P. Holl, M. Huth, G. Lutz, J. Soltau, D. Steigenhöfer, H. Soltau,

L. Strüder, PNSensor GmbH (Germany)

9154 0J Monolithic CMOS imaging x-ray spectrometers [9154-43]

A. Kenter, R. Kraft, T. Gauron, S. S. Murray, Smithsonian Astrophysical Observatory, HarvardSmithsonian Ctr. for Astrophysics (United States)

\section{SESSION $5 \quad$ IR DETECTORS I}

$9154 \mathrm{OL} \quad$ Asymmetry in the noise equivalent angle performance of the JWST fine guidance sensor [9154-10]

N. Rowlands, G. Warner, COM DEV Canada (Canada); L. Albert, Univ. de Montréal (Canada); T. Hardy, National Research Council Canada (Canada); J. Pipher, Univ. of Rochester (United States); J. Hutchings, National Research Council Canada (Canada); R. Doyon, Univ. de Montréal (Canada)

\section{SESSION 6 MANUFACTURER'S PROGRAMS}

915400 Characterisation activities of new NIR to VLWIR detectors from Selex ES Ltd at the UK ATC [9154-12]

N. Bezawada, D. Atkinson, UK Astronomy Technology Ctr. (United Kingdom); N. Shorrocks, L. Hipwood, H. Weller, SELEX ES Ltd. (United Kingdom); I. Bryson, UK Astronomy Technology Ctr. (United Kingdom); M. Jackson, R. Davis, K. Barnes, I. Baker, SELEX ES Ltd. (United Kingdom)

\section{SESSION 7 CCD AND CMOS DETECTORS}

9154 OP Assessment of the performance and radiation damage effects under cryogenic temperatures of a P-channel CCD204s [9154-99] N. J. Murray, A. D. Holland, J. P. D. Gow, D. J. Hall, K. D. Stefanov, B. J. Dryer, S. Barber, The Open Univ. (United Kingdom); D. J. Burt, e2v technologies plc (United Kingdom) 
$91540 Q \quad$ LGSD/NGSD: high speed optical CMOS imagers for E-ELT adaptive optics [9154-41] M. Downing, J. Kolb, European Southern Observatory (Germany); P. Balard, Lab. d'Astrophysique de Marseille (France); B. Dierickx, A. Defernez, Caeleste (Belgium); P. Feautrier, Institut de Planétologie et d'Astrophysique de Grenoble (France); G. Finger, European Southern Observatory (Germany); M. Fryer, e2v technologies plc (United Kingdom); J.-L. Gach, Lab. d'Astrophysique de Marseille (France); C. Guillaume, Observatoire de Haute-Provence (France); N. Hubin, European Southern Observatory (Germany); P. Jerram, P. Jorden, e2v technologies plc (United Kingdom); M. Meyer, European Southern Observatory (Germany); A. Payne, A. Pike, e2v technologies plc (United Kingdom); J. Reyes, European Southern Observatory (Germany); R. Simpson, e2v technologies plc (United Kingdom); E. Stadler, Institut de Planétologie et d'Astrophysique de Grenoble (France); J. Stent, N. Swift, e2v technologies plc (United Kingdom)

\section{SESSION 8 MISSIONS AND CAMERAS}

9154 OR The Euclid VIS CCD detector design, development, and programme status [9154-2] A. D. Short, European Space Research and Technology Ctr. (Netherlands); D. Barry, e2v technologies plc (United Kingdom); M. Berthe, Commissariat à l'Énergie Atomique (France); N. Boudin, European Space Research and Technology Ctr. (Netherlands); O. Boulade, Commissariat à l'Énergie Atomique (France); R. Cole, M. Cropper, Univ. College London (United Kingdom); L. Duvet, European Space Research and Technology Ctr. (Netherlands); J. Endicott, e2v technologies plc (United Kingdom); L. Gaspar Venancio, European Space Research and Technology Ctr. (Netherlands); J. Gow, The Open Univ. (United Kingdom); P. Guttridge, Univ. College London (United Kingdom); D. Hall, A. Holland, The Open Univ. (United Kingdom); H. Israel, Durham Univ. (United Kingdom); R. Kohley, European Space Astronomy Ctr. (Spain); R. Laureijs, J. Lorenzo Alvarez, European Space Research and Technology Ctr. (Netherlands); J. Martignac, Commissariat à l'Énergie Atomique (France); J. Maskell, e2v technologies plc (United Kingdom); R. Massey, Durham Univ. (United Kingdom); N. Murray, The Open Univ. (United Kingdom); S.-M. Niemi, Univ. College London (United Kingdom); P. Pool, e2v technologies plc (United Kingdom); S. Pottinger, Univ. College London (United Kingdom); T. Prod'homme, G. Racca, J.-C. Salvignol, European Space Research and Technology Ctr. (Netherlands); W. Suske, e2v technologies plc (United Kingdom); M. Szafraniec, Univ. College London (United Kingdom); P. Verhoeve, European Space Research and Technology Ctr. (Netherlands); D. Walton, Univ. College London (United Kingdom); R. Wheeler, e2v technologies plc (United Kingdom)

$91540 \mathrm{~V}$ Focal plane alignment and detector characterization for the Subaru prime focus spectrograph [9154-17]

M. Hart, R. H. Barkhouser, Johns Hopkins Univ. (United States); M. Carr, Princeton Univ. (United States); M. Golebiowski, Johns Hopkins Univ. (United States); J. E. Gunn, Princeton Univ. (United States); S. C. Hope, S. A. Smee, Johns Hopkins Univ. (United States)

\section{SESSION $9 \quad$ X-RAY DETECTORS II}

9154 OW The speedster-EXD: a new event-triggered hybrid CMOS $\mathbf{x}$-ray detector [9154-38] C. V. Griffith, A. D. Falcone, Z. R. Prieskorn, D. N. Burrows, The Pennsylvania State Univ. (United States) 
9154 OX A 3D CZT high resolution detector for $x$ - and gamma-ray astronomy [9154-37] I. Kuvvetli, C. Budtz-Jørgensen, DTU Space (Denmark); A. Zappettini, N. Zambelli, G. Benassi, Istituto dei Materiali per l'Elettronica ed il Magnetismo, CNR (Italy); E. Kalemci, Sabanci Univ. (Turkey); E. Caroli, J. B. Stephen, N. Auricchio, INAF - IASF Bologna (Italy)

9154 OY Caliste-SO: the x-ray spectrometer unit of the STIX instrument onboard the Solar Orbiter space mission [9154-36]

A. Meuris, O. Limousin, O. Gevin, CEA-IRFU (France); M.-C. Vassal, F. Soufflet, N. Fiant, 3D-Plus SAS (France); M. Bednarzik, C. Wild, S. Stutz, G. Birrer, Paul Scherrer Institut (Switzerland); C. Blondel, I. Le Mer, D.-D. Huynh, M. Donati, CEA-IRFU (France); O. Grimm, V. Commichau, ETH Zürich (Switzerland); G. Hurford, S. Krucker, Fachhochschule Nordwestschweiz (Switzerland); F. Gonzalez, M. Billot, Ctr. National d'Études Spatiales (France)

915410 Soft x-ray quantum efficiency of silicon hybrid CMOS detectors [9154-34]

Z. R. Prieskorn, The Pennsylvania State Univ. (United States); S. D. Bongiorno, Johns Hopkins Univ. (United States); D. N. Burrows, A. D. Falcone, C. V. Griffith, J. Nikoleyczik, The Pennsylvania State Univ. (United States)

\section{SESSION 10 BACK ILLUMINATION}

915411 Silicon sensor quantum efficiency, reflectance, and calibration [9154-18] M. Lesser, The Univ. of Arizona (United States)

915412 Enhancing the far-UV sensitivity of silicon CMOS imaging arrays [9154-40] K. D. Retherford, Southwest Research Institute (United States); Y. Bai, Teledyne Imaging Sensors (United States); K. K. Ryu, J. A. Gregory, MIT Lincoln Lab. (United States); P. B. Welander, MIT Lincoln Lab. (United States) and SLAC National Accelerator Lab. (United States); M. W. Davis, T. K. Greathouse, G. S. Winter, Southwest Research Institute (United States); V. Suntharalingam, MIT Lincoln Lab. (United States); J. W. Beletic, Teledyne Imaging Sensors (United States)

915413 Superlattice-doped silicon detectors: progress and prospects [9154-3] M. E. Hoenk, S. Nikzad, A. G. Carver, T. J. Jones, J. Hennessy, A. D. Jewell, Jet Propulsion Lab. (United States); J. Sgro, Alacron, Inc. (United States); S. Tsur, Applied Materials, Inc. (Israel); M. McClish, R. Farrell, Radiation Monitoring Devices, Inc. (United States)

\section{SESSION 11 LABORATORY SIMULATION}

915414 Laboratory simulation of Euclid-like sky images to study the impact of CCD radiation damage on weak gravitational lensing [9154-52] T. Prod'homme, P. Verhoeve, T. Oosterbroek, N. Boudin, A. Short, European Space Agency (Netherlands); R. Kohley, European Space Agency (Spain) 
915415 LSST optical beam simulator [9154-67]

J. A. Tyson, Univ. of California, Davis (United States); J. Sasian, College of Optical Sciences, The Univ. of Arizona (United States); K. Gilmore, SLAC National Accelerator Lab. (United States); A. Bradshaw, Univ. of California, Davis (United States); C. Claver, LSST Corp. (United States); M. Klint, Univ. of California, Davis (United States); G. Muller, GMTO Corp. (United States); G. Poczulp, National Optical Astronomy Observatory (United States); E. Resseguie, Univ. of California, Davis (United States)

\section{SESSION 12 TEST AND CHARACTERIZATION}

915416 CCD characterization for astronomy space missions at ESA [9154-15]

P. Verhoeve, T. Prod'homme, T. Oosterbroek, N. Boudin, L. Duvet, European Space Research and Technology Ctr. (Netherlands)

915417 Bad pixel mapping [9154-16]

R. M. Smith, D. Hale, Caltech Optical Obervatories, California Institute of Technology (United States); P. Wizinowich, W. M. Keck Observatory (United States)

915418 Electro-optical testing of fully depleted CCD image sensors for the Large Synoptic Survey Telescope camera [9154-26]

P. E. Doherty, Harvard Univ. (United States); P. Antilogus, P. Astier, Institut National de Physique Nucléaire et de Physique des Particules (France); J. Chiang, D. K. Gilmore, SLAC National Accelerator Lab. (United States); A. Guyonnet, Institut National de Physique Nucléaire et de Physique des Particules (France); D. Huang, Brookhaven National Lab. (United States); H. Kelly, SLAC National Accelerator Lab. (United States); I. Kotov, Brookhaven National Lab. (United States); P. Kubanek, Institute of Physics of the ASCR, v.v.i. (Czech Republic); A. Nomerotski, P. O'Connor, Brookhaven National Lab. (United States); A. Rasmussen, SLAC National Accelerator Lab. (United States); V. J. Riot, Lawrence Livermore National Lab. (United States); C. W. Stubbs, Harvard Univ. (United States); P. Takacs, Brookhaven National Lab. (United States); J. A. Tyson, Univ. of California, Davis (United States); K. Vetter, Brookhaven National Lab. (United States)

915419 Observatory deployment and characterization of SAPHIRA HgCdTe APD arrays [9154-1 1] D. Atkinson, D. Hall, C. Baranec, Univ. of Hawai'i (United States); I. Baker, SELEX ES Ltd. (United Kingdom); S. Jacobson, Univ. of Hawaili (United States); R. Riddle, California Institute of Technology (United States)

$91541 \mathrm{~A}$ Modelling and testing the x-ray performance of CCD and CMOS APS detectors using numerical finite element simulations [9154-27]

D. P. Weatherill, K. D. Stefanov, The Open Univ. (United Kingdom); T. A. Greig, e2v technologies plc (United Kingdom); A. D. Holland, The Open Univ. (United Kingdom) 


\section{Part Two}

\section{SESSION 13 ELECTRONICS AND CAMERAS}

9154 1B Impact of common modes correlations and time sampling on the total noise of a H2RG near-IR detector [9154-21]

B. Kubik, R. Barbier, A. Castera, E. Chabanat, S. Ferriol, G. Smadja, Institut de Physique Nucléaire de Lyon, CNRS, Univ. de Lyon (France)

9154 1C Performance of front-end mixed-signal ASIC for onboard CCD cameras [9154-22] H. Nakajima, S. Inoue, R. Nagino, N. Anabuki, K. Hayashida, H. Tsunemi, Osaka Univ. (Japan); J. P. Doty, Noqsi Aerospace, Ltd. (United States); H. Ikeda, Japan Aerospace Exploration Agency (Japan)

9154 1D Status of the NectarCAM camera project [9154-24]

J.-F. Glicenstein, CEA-IRFU (France); M. Barcelo, Institut de Física d'Altes Energies (Spain); J.-A. Barrio, Univ. Complutense de Madrid (Spain); O. Blanch, J. Boix, Institut de Física d'Altes Energies (Spain); J. Bolmont, Lab. de Physique Nucléaire et de Hautes Energies, IN2P3, CNRS (France) and Univ. Pierre et Marie Curie/Univ. Paris Diderot (France); C. Boutonnet, AstroParticule et Cosmologie, IN2P3, CNRS (France); P. Brun, Lab. Univers et Particules de Montpellier, IN2P3, CNRS (France); E. Chabanne, Lab. d'Annecy-le-Vieux de Physique des Particules, IN2P3, CNRS (France); C. Champion, S. Colonges, AstroParticule et Cosmologie, IN2P3, CNRS (France); P. Corona, Lab. de Physique Nucléaire et de Hautes Energies, IN2P3, CNRS (France) and Univ. Pierre et Marie Curie/Univ. Paris Diderot (France); B. Courty, AstroParticule et Cosmologie, IN2P3, CNRS (France); E. Delagnes, CEA-IRFU (France); C. Delgado, C. Diaz, Ctr. de Investigaciones Energéticas, Medioambientales y Tecnológicas (Spain); J.-P. Ernenwein, Ctr. de Physique des Particules de Marseille, IN2P3, CNRS (France); S. Fegan, O. Ferreira, Lab. Leprince-Ringuet, IN2P3, CNRS (France); M. Fesquet, CEA-IRFU (France); G. Fontaine, Lab. Leprince-Ringuet, IN2P3, CNRS (France); N. Fouque, Lab. d'Annecy-le-Vieux de Physique des Particules, IN2P3, CNRS (France); F. Henault, Institut de Planétologie et d'Astrophysique de Grenoble, INSU, CNRS (France); D. Gascón, Univ. de Barcelona (Spain); B. Giebels, Lab. Leprince-Ringuet, IN2P3, CNRS (France); D. Herranz, Univ. Complutense de Madrid (Spain); R. Hermel, Lab. d'Annecy-leVieux de Physique des Particules, IN2P3, CNRS (France); D. Hoffmann, Ctr. de Physique des Particules de Marseille, IN2P3, CNRS (France); D. Horan, Lab. Leprince-Ringuet, IN2P3, CNRS (France); J. Houles, Ctr. de Physique des Particules de Marseille, IN2P3, CNRS (France); P. Jean, Institut de Recherche en Astrophysique et Planétologie, INSU, CNRS (France) S. Karkar, Lab. de Physique Nucléaire et de Hautes Energies, IN2P3, CNRS (France) and Univ. Pierre et Marie Curie/Univ. Paris Diderot (France); J. Knödlseder, Institut de Recherche en Astrophysique et Planétologie, INSU, CNRS (France) G. Martinez, Ctr. de Investigaciones Energéticas, Medioambientales y Tecnológicas (Spain); G. Lamanna, T. LeFlour, Lab. d'Annecy-le-Vieux de Physique des Particules, IN2P3, CNRS (France); A. Lévêque, Institut de Recherche en Astrophysique et Planétologie, INSU, CNRS (France) R. Lopez-Coto, Institut de Física d'Altes Energies (Spain); F. Louis, Y. Moudden, E. Moulin, CEA-IRFU (France); P. Nayman, Lab. de Physique Nucléaire et de Hautes Energies, IN2P3, CNRS (France) and Univ. Pierre et Marie Curie/Univ. Paris Diderot (France); F. Nunio, CEA-IRFU (France); J.-F. Olive, Institut de Recherche en Astrophysique et Planétologie, INSU, CNRS (France) J.-L. Panazol, Lab. d'Annecy-le-Vieux de Physique des Particules, IN2P3, CNRS (France); S. Pavy, Lab. Leprince-Ringuet, IN2P3, CNRS (France); P.-O. Petrucci, Institut de Planétologie et d'Astrophysique de Grenoble, INSU, CNRS (France); M. Punch, AstroParticule et Cosmologie, IN2P3, CNRS (France); J. Prast, Lab. d'Annecy-le-Vieux de 
Physique des Particules, IN2P3, CNRS (France); P. Ramon, Institut de Recherche en Astrophysique et Planétologie, INSU, CNRS (France) S. Rateau, Lab. Leprince-Ringuet, IN2P3, CNRS (France); M. Ribó, Univ. de Barcelona (Spain); S. Rosier-Lees, Lab. d'Annecyle-Vieux de Physique des Particules, IN2P3, CNRS (France); A. Sanuy, Univ. de Barcelona (Spain); P. Sizun, CEA-IRFU (France); J. Siero, Univ. de Barcelona (Spain); K.-H. Sulanke, Deutsches Elektronen-Synchrotron (Germany); J.-P. Tavernet, Lab. de Physique Nucléaire et de Hautes Energies, IN2P3, CNRS (France) and Univ. Pierre et Marie Curie/Univ. Paris Diderot (France); L. A. Tejedor, Univ. Complutense de Madrid (Spain); F. Toussenel, Lab. de Physique Nucléaire et de Hautes Energies, IN2P3, CNRS (France) and Univ. Pierre et Marie Curie/Univ. Paris Diderot (France); G. Vasileiadis, Lab. Univers et Particules de Montpellier, IN2P3, CNRS (France); V. Voisin, Lab. de Physique Nucléaire et de Hautes Energies, IN2P3, CNRS (France) and Univ. Pierre et Marie Curie/Univ. Paris Diderot (France); V. Waegebert, Institut de Recherche en Astrophysique et Planétologie, INSU, CNRS (France) C. Zurbach, Lab. Univers et Particules de Montpellier, IN2P3, CNRS (France)

$9154 \mathrm{IE}$ Technology and device-design enhancements for improved read noise performance in fully depleted CCDs [9154-25]

S. E. Holland, C. J. Bebek, Lawrence Berkeley National Lab. (United States); F. Dion, R. Frost, R. Groulx, Teledyne DALSA Semiconductor (Canada); J. S. Lee, G. Wang, Lawrence Berkeley National Lab. (United States)

\section{SESSION 14 IR DETECTORS II}

9154 IF Near-infrared InGaAs detectors for background-limited imaging and photometry [9154-76] P. W. Sullivan, B. Croll, R. A. Simcoe, MIT Kavli Institute for Astrophysics and Space Research (United States)

$91541 G$ Quantum efficiency test set up performances for NIR detector characterization at ESTEC [9154-45]

P.-E. Crouzet, L. Duvet, F. De Wit, T. Beaufort, S. Blommaert, B. Butler, G. Van Duinkerken, J. ter Haar, J. Heijnen, K. van der Luijt, H. Smit, European Space Research and Technology Ctr. (Netherlands); T. Viale, ONERA (France)

$91541 \mathrm{~J}$ AQUARIUS: the next generation mid-IR detector for ground-based astronomy, an update. [9154-48]

D. Ives, G. Finger, G. Jakob, European Southern Observatory (Germany); U. Beckmann, Max-Planck-Institut für Radioastronomie (Germany)

POSTERS: MONDAY

$91541 \mathrm{~K} \quad$ Pocket pumped image analysis [9154-49]

I. Kotov, P. O'Connor, Brookhaven National Lab. (United States); N. Murray, The Open Univ. (United Kingdom)

$91541 \mathrm{~L}$ Optimization and performance of H2RG detectors and SIDECAR ASICs for SWIMS [9154-50] S. Todo, K. Tateuchi, K. Motohara, M. Konishi, H. Takahashi, Y. Kitagawa, N. M. Kato, The Univ. of Tokyo (Japan) 
9154 IM ESO adaptive optics NGSD/LGSD prototype controller for the E-ELT [9154-51]

J. Reyes, M. Downing, European Southern Observatory (Germany); J. Romero, Univ. de Málaga (Spain)

$91541 \mathrm{~N}$ High event rate ROICs (HEROICs) for astronomical UV photon counting detectors [9154-53] A. Harwit, Ball Aerospace \& Technologies Corp. (United States); K. France, Univ. of Colorado at Boulder (United States); V. Argabright, S. Franka, E. Freymiller, D. Ebbets, Ball Aerospace \& Technologies Corp. (United States)

915410 Evaluation of InGaAs $640 \times 512$ detector array manufactured by Chunghwa Leading Photonics Tech [9154-54]

T. Nagayama, Nagoya Univ. (Japan) and Kagoshima Univ. (Japan); N. Takeuchi, T. Kokusho, A. Yamanaka, M. Nishiyama, H. Kaneda, Nagoya Univ. (Japan)

$91541 P \quad$ Driving a CCD with two ASICs: CABAC and ASPIC [9154-55]

C. Juramy, P. Antilogus, P. Bailly, S. Baumont, M. Dhellot, Lab. de Physique Nucléaire et de Hautes Énergies (France); M. El Berni, J. Jeglot, Lab. de l'Accélérateur Linéaire, CNRS, Ctr. Scientifique d'Orsay (France); H. Lebbolo, D. Martin, Lab. de Physique Nucléaire et de Hautes Énergies (France); A. Qureshi, Lab. de l'Accélérateur Linéaire, CNRS, Ctr.

Scientifique d'Orsay (France); S. Russo, D. Terront, Lab. de Physique Nucléaire et de Hautes Énergies (France); V. Tocut, P. Vallerand, Lab. de l'Accélérateur Linéaire, CNRS, Ctr. Scientifique d'Orsay (France)

$91541 Q \quad$ Impact of noise covariance and nonlinearities in NIR H2RG detectors [9154-57] B. Kubik, R. Barbier, A. Castera, E. Chabanat, S. Ferriol, G. Smadja, Institut de Physique Nucléaire de Lyon, CNRS, Univ. de Lyon (France)

9154 IR SiPM detectors for the ASTRI project in the framework of the Cherenkov Telescope Array [9154-58]

S. Billotta, D. Marano, G. Bonanno, M. Belluso, A. Grillo, S. Garozzo, G. Romeo, M. C. Timpanaro, INAF - Osservatorio Astrofisico di Catania (Italy); M. C. Maccarone, O. Catalano, G. La Rosa, G. Sottile, D. Impiombato, C. Gargano, S. Giarrusso, INAF - Istituto di Astrofisica Spaziale e Fisica Cosmica di Palermo (Italy)

9154 is Development of a vacuum UV CCD system for spectrograph testing [9154-61] Q. Song, Z. Zhao, W. Wang, National Astronomical Observatories (China)

9154 1T A new method of CCD dark current correction via extracting the dark Information from scientific images [9154-62]

B. Ma, National Astronomical Observatories (China); Z. Shang, Tianjin Normal Univ. (China) and National Astronomical Observatories (China); Y. Hu, Q. Liu, National Astronomical Observatories (China); L. Wang, Purple Mountain Observatory (China) and Texas A\&M Univ. (United States); P. Wei, National Astronomical Observatories (China)

$91541 \mathrm{U}$ The nonlinear photon transfer curve of CCDs and its effects on photometry [9154-63] B. Ma, National Astronomical Observatories (China); Z. Shang, Tianjin Normal Univ. (China) and National Astronomical Observatories (China); L. Wang, Purple Mountain Observatory (China) and Texas A\&M Univ. (United States); Y. Hu, Q. Liu, P. Wei, National Astronomical Observatories (China)

$91541 \mathrm{~V} \quad$ Reducing noise from a Stirling micro cooler used with an InSb diode [9154-64]

N. R. Bingham, M. C. B. Ashley, The Univ. of New South Wales (Australia) 
$9154 \mathrm{IW}$ Measurement of pixel response functions of a fully depleted CCD [9154-65]

Y. Kobayashi, National Astronomical Observatory of Japan (Japan), The Univ. of Tokyo (Japan), and The Graduate Univ. for Advanced Studies (Japan); Y. Niwa, National Astronomical Observatory of Japan (Japan); T. Yano, National Astronomical Observatory of Japan (Japan) and The Graduate Univ. for Advanced Studies (Japan); N. Gouda, National Astronomical Observatory of Japan (Japan), The Univ. of Tokyo (Japan), and The Graduate Univ. for Advanced Studies (Japan); T. Hara, The Univ. of Tokyo (Japan);

Y. Yamada, Kyoto Univ. (Japan)

$91541 \mathrm{X}$ Characterization and optimization for detector systems of IGRINS [9154-66]

U. Jeong, M.-Y. Chun, J. S. Oh, C. Park, I.-S. Yuk, H. Oh, K.-M. Kim, K. Y. Ko, Korea Astronomy and Space Science Institute (Korea, Republic of); M. Pavel, The Univ. of Texas at Austin (United States); Y. S. Yu, Korea Astronomy and Space Science Institute (Korea, Republic of); D. T. Jaffe, The Univ. of Texas at Austin (United States)

9154 1Y A new technique of characterization of the intrapixel response of astronomical detectors [9154-68]

C. Ketchazo, CEA-Ctr. de SACLAY (France); T. Viale, ONERA (France); O. Boulade, CEA-Ctr. de SACLAY (France); G. Druart, ONERA (France); V. Moreau, CEA-Ctr. de SACLAY (France): L. Mugnier, ONERA (France); D. Dubreuil, CEA-Ctr. de SACLAY (France); S. Derelle, ONERA (France); S. Ronayette, CEA-Ctr. de SACLAY (France); N. Guérineau, ONERA (France); M. Berthe, CEA-Ctr. de SACLAY (France)

915412 A few phenomena on the fully depleted CCDs at the engineering observation of Hyper Suprime-Cam [9154-69]

Y. Kamata, H. Nakaya, S. Kawanomoto, S. Miyazaki, National Astronomical Observatory of Japan (Japan)

915421 Development of an ASIC for the readout and control of near-infrared large array detectors [9154-71]

D. Meier, H. K. O. Berge, A. Hasanbegovic, M. A. Altan, B. Najafiuchevler, S. Azman, J. Talebi, A. Olsen, P. Øya, P. Paahlsson, C. Gheorghe, G. Maehlum, Integrated Detector Electronics AS (Norway)

915422 Development of a stacked defector system for the $\mathrm{x}$-ray range and its possible applications [9154-72]

D. Maier, Institut für Astronomie und Astrophysik, Eberhard Karls Univ. Tübingen (Germany);

O. Limousin, A. Meuris, Commissariat à l'Énergie Atomique (France); S. Pürckhaver, A. Santangelo, T. Schanz, C. Tenzer, Institut für Astronomie und Astrophysik Eberhard Karls Univ. Tübingen (Germany)

915424 Design of the front end electronics for the infrared camera of JEM-EUSO, and manufacturing and verification of the prototype model [9154-75]

O. Maroto, L. Diez-Merino, J. Carbonell, A. Tomàs, NTE--SENER S.A. (Spain); M. Reyes, E. Joven, Y. Martín, Instituto de Astrofísica de Canarias (Spain); J. A. Morales de los Ríos, L. del Peral, Univ. de Alcalá (Spain); M. D. Rodríguez-Frías, Univ. de Alcalá (Spain) and Instituto de Astrofísica de Canarias (Spain)

915425 Finite-differences model to predict temperatures on cryogenic focal plane arrays: first laboratory results [9154-77]

D. Guzmán, R. Bilbeny, Pontificia Univ. Católica de Chile (Chile); A. Szentgyorgyi,

T. J. Norton, Harvard-Smithsonian Ctr. for Astrophysics (United States) 
915426 SIDECAR ASIC firmware for astronomy applications [9154-78]

J. Chen, Teledyne Imaging Sensors (United States); M. Loose, Markury Scientific, Inc. (United States); R. Ricardo, J. Beletic, M. Farris, M. Xu, A. Wong, C. Cabelli, Teledyne Imaging Sensors (United States)

915427 System for photomultiplier tubes characterization and data acquisition for water Cherenkov detectors [9154-79]

M. J. Bonilla Rosales, E. Carrasco, I. Torres, Instituto Nacional de Astrofísica, Óptica y Electrónica (Mexico); E. Moreno, Benemérita Univ. Autónoma de Puebla (Mexico); A. Carramiñana, Instituto Nacional de Astrofísica, Óptica y Electrónica (Mexico)

915428 Using dummy and pseudo-dummy amplifiers to correct for common mode CCD noise [9154-80]

I. A. Steele, C. J. Mottram, R. J. Smith, R. M. Barnsley, Liverpool John Moores Univ. (United Kingdom)

9154 2A Cosmic ray response of megapixel LWIR arrays from TIS [9154-82]

J. J. Girard, Univ. of Rochester (United States) and Siena College (United States);

W. J. Forrest, C. W. McMurtry, J. L. Pipher, M. Dorn, Univ. of Rochester (United States);

A. Mainzer, Jet Propulsion Lab. (United States)

$91542 \mathrm{C}$ Candidate detector assessment for the CASTOR mission [9154-84]

A. D. Scott, COM DEV International Ltd. (Canada); P. Cote, NRC - Herzburg Institute of Astrophysics (Canada); N. Rowlands, COM DEV International Ltd. (Canada); O. Daigle, Nüvü Camēras Inc. (Canada)

$91542 \mathrm{D}$ Intra-pixel response of the new JWST infrared detector arrays [9154-85]

T. Hardy, C. Willot, J. Pazder, National Research Council Canada (Canada)

91542 G CCD readout electronics for the Subaru Prime Focus Spectrograph [9154-88]

S. C. Hope, Johns Hopkins Univ. (United States); J. E. Gunn, C. P. Loomis, Princeton Univ. (United States); R. E. Fitzgerald, FitzGerald Engineering (United States); G. O. Peacock, Johns Hopkins Univ. (United States)

$91542 \mathrm{H} \quad$ Progress in development of H4RG-10 infrared focal plane arrays for WFIRST-AFTA [9154-89] E. C. Piquette, W. McLevige, J. Auyeung, A. Wong, Teledyne Imaging Sensors (United States)

91542 Characteristic of e2v CMOS sensors for astronomical applications [9154-90] S.-Y. Wang, H.-H. Ling, Y.-S. Hu, Institute of Astronomy and Astrophysics, Academia Sinica (Taiwan); J. C. Geary, S. M. Amato, Harvard-Smithsonian Ctr. for Astrophysics (United States); J. Pratlong, A. Pike, P. Jordan, e2v technologies plc (United Kingdom); M. J. Lehner, Institute of Astronomy and Astrophysics, Academia Sinica (Taiwan)

$91542 \mathrm{~J}$ Electronics design for a high precision image stabilization system [9154-91]

A. Casas, D. Roma, M. Carmona, J. M. Gómez, J. Bosch, M. López, J. Sabater, A. Herms, Univ. de Barcelona (Spain); T. Mave, E. Nakai, R. Volkmer, W. Schmidt, Kiepenhever-Institut für Sonnenphysik (Germany) 
$91542 \mathrm{~K}$ On the calibration of a single channel cosmic ray particle detector [9154-92]

A. H. Maghrabi, A. S. Alghamdi, King Abdulaziz City for Science and Technology (Saudi Arabia); R. Alotaibi, King Saud Univ. (Saudi Arabia); M. M. Almutari, King Abdulaziz City for Science and Technology (Saudi Arabia); M. S. Garawi, King Saud Univ. (Saudi Arabia)

$91542 \mathrm{~L}$ Designing and constructing of a two scintillator crystal rotatable telescope for muon flux variation studies [9154-93]

A. Alghamdi, A. H. Maghrabi, M. M. Almutari, King Abdulaziz City for Science and Technology (Saudi Arabia)

9154 2N NASA's Physics of the Cosmos and Cosmic Origins technology development programs [9154-96]

M. Clampin, T. Pham, NASA Goddard Space Flight Ctr (United States)

915420 A calibration method for the measurement of IR detector spectral responses using a FTIR spectrometer equipped with a DTGS reference cell [9154-97]

O. Gravrand, J. Wlassow, L. Bonnefond, CEA-LETI MINATEC (France)

Author Index 


\title{
Conference Committee
}

\author{
Symposium Chairs
}

Gillian S. Wright, UK Astronomy Technology Centre (United Kingdom) Luc Simard, National Research Council Canada (Canada)

Symposium Co-chairs

Colin Cunningham, UK Astronomy Technology Centre (United Kingdom)

Masanori lye, National Astronomical Observatory of Japan (Japan)

Conference Chairs

Andrew D. Holland, e2v Center for Electronic Imaging at The Open University (United Kingdom)

James W. Beletic, Teledyne Imaging Sensors (United States)

Conference Program Committee

Megan E. Eckart, NASA Goddard Space Flight Center (United States) Gert Finger, European Southern Observatory (Germany)

Paul Jorden, e2v technologies plc (United Kingdom)

Didier D. Martin, European Space Research and Technology Centre (Netherlands)

Satoshi Miyazaki, National Astronomical Observatory of Japan (Japan)

Peter C. Moore, National Optical Astronomy Observatory (United States)

S. Harvey Moseley, NASA Goddard Space Flight Center (United States)

Robert H. Philbrick, Ball Aerospace \& Technologies Corporation (United States)

Roger M. Smith, California Institute of Technology (United States) Lothar Strüder, PNSensor GmbH (Germany)

Tadayuki Takahashi, Japan Aerospace Exploration Agency (Japan) Hiroshi Tsunemi, Osaka University (Japan)

\section{Session Chairs}

1 Programs

Andrew D. Holland, e2v Center for Electronic Imaging at The Open University (United Kingdom) 
Radiation Damage

Andrew D. Holland, e2v Center for Electronic Imaging at The Open University (United Kingdom)

3 EM-CCDs

Satoshi Miyazaki, National Astronomical Observatory of Japan (Japan)

4 X-ray Detectors I

Paul Jorden, e2v technologies plc (United Kingdom)

5 |R Detectors |

Paul Jorden, e2v technologies plc (United Kingdom)

6 Manufacturer's Programs

Peter Verhoeve, European Space Research and Technology Centre (Netherlands)

$7 \quad$ CCD and CMOS Detectors

Peter Verhoeve, European Space Research and Technology Centre (Netherlands)

8 Missions and Cameras

Megan E. Eckart, NASA Goddard Space Flight Center (United States)

9 X-ray Detectors II

Megan E. Eckart, NASA Goddard Space Flight Center (United States)

10 Back lllumination

Roger M. Smith, California Institute of Technology (United States)

11 Laboratory Simulation

Roger M. Smith, California Institute of Technology (United States)

12 Test and Characterization

Satoshi Miyazaki, National Astronomical Observatory of Japan (Japan)

13 Electronics and Cameras

James W. Beletic, Teledyne Imaging Sensors (United States)

14 IR Detectors II

Gert Finger, European Southern Observatory (Germany) 